\title{
Slow reacquisition of a conditioned taste aversion
}

\author{
JULIE A. HART, MARK J. BOURNE, and TODD R. SCHACHTMAN \\ University of Missouri, Columbia, Missouri
}

\begin{abstract}
Rats were used to examine the extent to which extinction of an acquired conditioned taste aversion retards subsequent reacquisition. A saccharin-flavored solution (sac) was paired with $\mathrm{LiCl}$ and then followed by CS-alone extinction trials with this flavor. A control group received a different flavor, decaffeinated-coffee (coff), during initial conditioning and extinction. Sac was then paired with $\mathrm{LiCl}$ for all rats during a second conditioning phase. Reacquisition of the aversion to sac was retarded relative to the acquisition of an aversion to sac by the control group. A similar experiment with fewer extinction trials, but still with complete loss of the initial aversion, did not obtain slow reacquisition. The results are discussed with respect to an interference view of extinction and the slowreacquisition effect.
\end{abstract}

Recent studies have examined the extent to which conditioned responding is reacquired when an extinguished conditioned stimulus (CS) is again paired with the unconditioned stimulus (US). Bouton (1986), using a conditioned emotional response (CER) procedure with rats, paired a tone with footshock during initial conditioning. After numerous tone-alone extinction trials, the tone was again paired with footshock, and the conditioned response (CR) was assessed. The rats reacquired the CR slowly in comparison with a group that received tone-footshock pairings for the first time during the latter conditioning phase (see also Bouton \& Swartzentruber, 1989). This slow reacquisition effect occurred when the CS was given extensive extinction treatment (i.e., 72 trials). A less surprising "savings" effect-namely, more rapid reacquisition of an extinguished conditioned response-occurred with less extinction treatment (i.e., 16 trials).

More recently, Napier, Macrae, and Kehoe (1992) used a nictitating membrane response (NMR) procedure with rabbits to examine the rate of reacquisition of an extinguished response. The experiments by Napier et al. did not provide any evidence of slow reacquisition of the $\mathrm{CR}$, despite the use of extensive extinction prior to reconditioning. Reacquisition of the CS in these experiments uniformly produced a savings effect. Napier et al. suggested that slow reacquisition may be specific to certain conditioning preparations, such as conditioned suppression, but that it may not occur with other procedures, such as NMR preparation.

Support for this research came from funds provided by the Research Council of the University of Missouri-Columbia. We thank B. Butchart, J. Calton, K. Gustavson, B. Luce, M. McEwen, and K. Mitchell for critically reading a preliminary version of this manuscript and for help in conducting the experiments. We also thank $\mathbf{R}$. Miller for reading an early version of this manuscript. Address reprint requests to T. R. Schachtman, Department of Psychology, 210 McAlester Hall, University of Missouri, Columbia, MO 65211 .

-Accepted by previous editor, Vincent M. LoLordo
A distinction has been drawn between CER and NMR with respect to when slow reacquisition can be expected. Moreover, there have also been conflicting findings, even with the use of a single preparation. Although Danguir and Nicolaidis (1977), using conditioned taste aversion (CTA), obtained a slow-reacquisition effect that parallels the slow-reacquisition effects obtained by Bouton (1986) and Bouton and Swartzentruber (1989) with conditioned suppression, Revusky and Coombes (1979), on the other hand, stated that slow reacquisition does not occur with a CTA procedure. They claimed to have obtained little evidence of slow reacquisition of extinguished taste aversions, despite having varied several conditioning parameters.

The equivocal findings among studies examining reacquisition effects with a CTA procedure are especially surprising given that CTA possesses several features that make it ideal for obtaining slow reacquisition. Bouton (1986) and Napier et al. (1992) point out that slow reacquisition may be more likely when a long intertrial interval (ITI) is used during initial conditioning. When short ITIs are used, aftereffects from the US presentation on the previous trial become part of the stimulus context for the subsequent trial. These cues would not extinguish during the CS-alone presentations, and could contribute to a strong $C R$ during reacquisition (i.e., by producing a semblance of rapid reacquisition). The long ITIs commonly used during CTA conditioning minimize the influence of these US aftereffects during conditioning. In fact, the use of a single conditioning trial, as used for initial conditioning in the present study, necessarily eliminates these effects. Moreover, both Bouton and Napier et al. state that spontaneous recovery can also attentuate the slow-reacquisition effect. Spontaneous recovery has been noticeably absent in many CTA studies, including recent ones using intervals as long as 3 and 21 days (Kraemer \& Spear, 1992; Schachtman, Brown, \& Miller, 1985), as well as in unpublished data from our laboratory employing highly similar parameters to those used in the present study. It is impor- 
tant to use a procedure that produces little spontaneous recovery between sessions, since this recovery will work against obtaining a slow-reacquisition effect. Using treatments aimed at minimizing the effects of spontaneous recovery and US aftereffects, the present experiments examined the potential for slow reacquisition using a CTA procedure.

\section{EXPERIMENT 1}

In Experiment 1 , all subjects initially received a single exposure to a flavored solution, which was followed by $\mathrm{LiCl}$-induced illness. They all then received three extinction trials with this flavor. For half of the subjects (Group Reacqu), this flavor was saccharin (sac), and for the other subjects (Group Control), the flavor was coffee (coff). After this extinction treatment, all animals were then given one conditioning trial. This was a reacquisition trial for Group Reacqu. If extinction produces slow reacquisition, Group Control should exhibit a greater aversion to sac than Group Reacqu.

\section{Method}

Subjects. For Experiment 1, the subjects were four female and six male Sprague-Dawley-derived rats bred in the University of Missouri colony. At the start of the study, the female and male rats weighed $255-270 \mathrm{~g}$ and $301-425 \mathrm{~g}$, respectively. Each rat was individually housed in a standard hanging, stainless-steel, wire-mesh cage with ad-lib access to lab chow. The subjects were gradually water deprived prior to the start of the experiment, culminating in $10 \mathrm{~min}$ of water access each day. Water access occurred in the home cage after each day's experimental manipulations, approximately $23 \mathrm{~h}$ prior to the experimental manipulations of the next day. The room housing the animals was on a 16:8-h, light:dark cycle, and treatments occurred during the middle of the light portion of the cycle.

Apparatus. All treatments occurred in the home cage. A clip on the front of each cage could hold a plastic drinking tube (a modified, inverted, $50-\mathrm{ml}$ syringe with a metal lick tube attached). $\mathrm{LiCl}$ was administered using a $25-\mathrm{ga}, 1.59-\mathrm{cm}$ hypodermic needle.

Procedure. The subjects were divided into two groups, counterbalanced as much as possible for body weight and sex. Four of the animals were assigned to Group Control, and the remaining animals were assigned to Group Reacqu. On Day 1 , subjects in Group Reacqu received a 10 -min exposure to a $0.1 \%(w / v)$ saccharin solution (Sigma, St. Louis, MO), designated "sac," while Group Control received a 10-min exposure to a 3\% (w/v) decaffeinated-coffee (Sanka; General Foods, White Plains, NY) solution, designated "coff." Throughout the experiment, intake of all flavored solutions given during experimental treatments was recorded by weighing the drinking tubes before and after treatment. Immediately after exposure to the solution, each animal received an intraperitoneal injection of $2 \%(\mathrm{w} / \mathrm{v}) \mathrm{LiCl}$ at $1 \%$ body weight (i.e., $200 \mathrm{mg} / \mathrm{kg}$ ). On Day 2, the animals were given no treatments, except their daily 10-min exposure to water, to ensure recovery from illness. In this, and all subsequent, experiments, if an animal did not drink $2 \mathrm{ml}$ or more of the target solution during the initial exposure to the solution (i.e., when the solution had not yet been paired with $\mathrm{LiCl}$ ), it was eliminated from the experiment due to insufficient CS exposure. In practice, no animals in Experiment 1 failed to meet this criterion.

Extinction trials occurred on Days 3, 4, and 5. The subjects in Group Reacqu received daily $10-\mathrm{min}$ exposures to sac, while the rats in Group Control were given coff for $10 \mathrm{~min}$. To make the present procedure comparable with planned experiments, no treatments were given on Days 6 and 7 . These days were included to make the procedure similar to that of planned experiments which would give treatments on these days. On Day 8 , all subjects received a 10-min exposure to sac, followed by an injection of $\mathrm{LiCl}$ exactly like the one given on Day 1. On Day 9, the animals were given no treatments, except their daily 10 -min exposure to water. All subjects were given a 10-min exposure to sac during testing on each of Days 10-12.

\section{Results and Discussion}

In general, the results yielded no slow-reacquisition effect when only three extinction trials were given. For all analyses of variance (ANOVAs), the criterion for statistical significance was $p<.05$. An ANOVA conducted on the data from the Day 1 conditioning trial revealed group differences. Subjects consumed more sac than coff $[F(1,8)=9.99]$. Both groups revealed an aversion as demonstrated by a decrease in consumption on the initial extinction trial relative to Day 1 consumption, as revealed by a main effect of day $[F(1,8)=61.41]$. Since more sac than coff was consumed, there was a main effect of group $[F(1,8)=14.24]$, but no interaction was obtained $(F<1)$. A similar statistical pattern occurred across the extinction trials. An ANOVA on these data yielded a main effect of day, showing that the groups increased their consumption across extinction trials $[F(2,16)=13.14]$. There was also a main effect of group, since sac was consumed at a higher level than coff $[F(1,8)=24.10]$, but no interaction was found $(F<1)$. The groups consumed similar amounts (group mean $\pm S E M$ ) of sac during the sac- $\mathrm{LiCl}$ trial following extinction on Day 8 [12.7 $( \pm 2.2) \mathrm{ml}$ for Group Reacqu and $11.0( \pm 0.4) \mathrm{ml}$ for Group Control; $F<1]$. This lack of a difference on the reacquisition trial shows that if any neophobia occurred for Group Control, it did not produce significantly lower consumption than that by Group Reacqu.

Group mean consumption of sac on the test trials on Days $10-12$ was $4.1( \pm 0.8) \mathrm{ml}, 6.0( \pm 1.3) \mathrm{ml}$, and 8.8 $( \pm 1.7) \mathrm{ml}$ for Group Reacqu and $4.7( \pm 1.4) \mathrm{ml}, 7.0$ $( \pm 1.4) \mathrm{ml}$, and $10.1( \pm 2.2) \mathrm{ml}$ for Group Control. An ANOVA (group $\times$ day) conducted on consumption during these test trials yielded no main effect of group, nor any group $\times$ day interaction $(F<1)$. The groups did increase their consumption across days $[F(2,16)=$ 32.72]. These results did not reveal slower reacquistion by Group Reacqu. There was also no evidence of a "savings" effect; that is, Group Reacqu did not reacquire the aversion to sac more quickly than Group Control learned about sac.

In Experiment 1, no evidence was obtained of slow reconditioning of an aversion following extinction. However, since only a single conditioning trial was used, reacquisition of the aversion may not have been as pronounced as it would have been if more reconditioning trials had been given. Group Control consumed $4.7 \mathrm{ml}$ on the first test trial, and it is possible that an additional reacquisition trial may have both increased the aversion for this 
condition and enhanced sensitivity for producing group differences. Moreover, only three extinction trials were used in Experiment 1, and, as indicated by previous studies examining the slow-reacquisition effect (e.g., Bouton, 1986), extensive extinction is necessary to observe slow reacquisition.

\section{EXPERIMENT 2}

In Experiment 2, we sought to determine whether a larger number of extinction trials, relative to the number used in Experiment 1, would produce the slowreacquisition effect. The procedure of Experiment 2 was very similar to that of Experiment 1, except as follows. In Experiment 2, nine extinction trials were used; this extensive extinction treatment was expected to be much greater than that needed to eliminate the CR to the flavor, and was expected to increase sensitivity to observing slow reacquisition. A low dose of $\mathrm{LiCl}$, relative to that used in Experiment 1, was used during initial conditioning to weaken the aversion, and thus increase the effectiveness of the extinction trials. In addition, two reacquisition trials were used in Experiment 2, as well as a lower dose of $\mathrm{LiCl}$ during reacquisition, relative to the dose used in Experiment 1. Two trials were used during this later conditioning phase, in order to produce a greater overall aversion than that produced in Experiment 1.

\section{Method}

Subjects and Apparatus. Seven female and eight male waterdeprived, Sprague-Dawley-derived rats served as subjects. The body weights of the female and male rats were, respectively, 247-365 g and 283-489 g. All rats were maintained, and all features of the apparatus were the same, as in Experiment 1.

Procedure. On Day 1, the rats were given $10 \mathrm{~min}$ of access to a lick tube containing either sac ( $n=8$, Group Reacqu) or coff ( $n=7$, Group Control), which was followed immediately by an injection of $0.3 \mathrm{M} \mathrm{LiCl}$ at $1.33 \%$ body weight. The subjects recovered from illness on Day 2, and on Days 3-11, they each received a 10-min exposure to the solution to which they had been exposed on Day 1 , but this time without $\mathrm{LiCl}$. No treatments were given on Days 12 and 13. On Days 14 and 16, all subjects received sac for $10 \mathrm{~min}$, followed by an injection of $0.15 \mathrm{M} \mathrm{LiCl}$ at $1 \%$ body weight.

On Days 15 and 17, the animals were given no treatments, except their daily 10 -min exposure to water, to allow recovery from $\mathrm{LiCl}$. All subjects were given a 10 -min exposure to sac during testing on each of Days 18-22. All details of the experiment that are unspecified here were unchanged from Experiment 1.

\section{Results and Discussion}

The overall results revealed that a slow-reacquisition effect occurred. One animal in Group Control was eliminated from the experiment because it failed to drink at least $2 \mathrm{ml}$ of sac during the initial exposure to this solution. An ANOVA conducted on the data from the Day 1 conditioning trial revealed group differences. The amount of sac consumed by Group Reacqu was greater than the amount of coff consumed by Group Control $[F(1,12)=$ 36.65]. Both groups experienced a decrease in consumption on the initial extinction trial relative to consumption on Day 1 , as revealed by a main effect of day $[F(1,12)=$
156.33], and since more sac than coff was consumed, there was a main effect of group, as well as a group $\times$ day interaction $(F \mathrm{~s}>33.97)$. A comparable pattern occurred on the extinction trials. An ANOVA conducted on the data from the first and last extinction trials produced a main effect of day, showing that the groups increased their consumption across extinction trials $[F(1,12)=$ $369.89]$. There was also a main effect of group, since sac was consumed at a higher level than coff $[F(1,12)=$ $12.07]$, as well as a group $\times$ day interaction $[F(1,12)=$ 6.44]. The different amounts consumed of the two flavors is awkward, but the treatment given Group Control served its purpose by providing these subjects and Group Reacqu with comparable exposure to $\mathrm{LiCl}$ and to the drinking tubes.

Groups Reacqu and Control consumed similar amounts of sac on the sac-LiCl trial on Day $14(F<1)$. This absence of a difference shows that any neophobia occurring for Group Control did not produce appreciably lower consumption by that group than by Group Reacqu. On Day 14, the groups consumed $16.5( \pm 2.4) \mathrm{ml}$ (Group Reacqu) and $17.8( \pm 1.6) \mathrm{ml}$ (Group Control).

Group mean consumption of sac on the conditioning trial on Day 16 revealed the effect of the conditioning trial on Day 14. A between-group difference in intake was observed on the Day 16 conditioning trial $[F(1,12)=$ 19.59]. Group Reacqu consumed $10.1( \pm 1.6) \mathrm{ml}$, and Group Control drank 1.6 $( \pm 0.6) \mathrm{ml}$ on Day 16. This difference indicated slow reacquisition by Group Reacqu.

Consumption of sac on the test trials on Days 18-22 is represented in Figure 1. An ANOVA conducted on these data yielded main effects of group $[F(1,12)=$ $5.56, p<.05]$ and of day $[F(1,4)=4.18, p<.01]$, but no group $\times$ day interaction occurred $[F(4,48)=2.52, p<$ $.055]$. These results revealed that the subjects in Group Reacqu consumed more sac at test than did the animals in Group Control.

This evidence indicates that an excitatory CS subsequently given extensive CS-alone extinction produces slow reacquisition of conditioning. The findings confirm the conclusions of Bouton (1986) and Bouton and

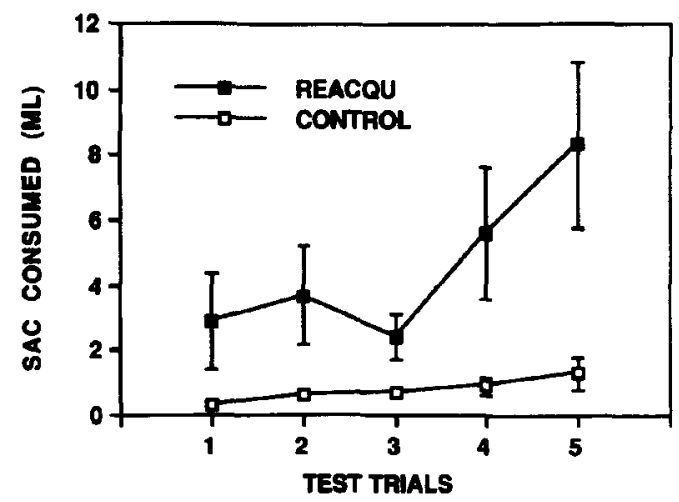

Figure 1. Mean intake ( $\pm S E M s)$ of a saccharin solution on the test trials in Experiment 2. 
Swartzentruber (1989) in their studies using a CER procedure, and corroborate the CTA results of Danguir and Nicolaidis (1977).

\section{EXPERIMENT 3}

The slow-reacquisition effect observed in Experiment 2 used sac as the target CS and coff as the control flavor. It is possible that the retarded excitatory conditioning seen after extensive extinction is specific to the flavors used in Experiment 2-namely, a saccharin flavor as the target CS and a coffee-flavored solution as the control CS. This possibility becomes more convincing when it is noted that Revusky and Coombes (1979, Experiment 2), despite their general conclusion that slow reacquisition does not occur with CTA conditioning, found some evidence of slow reacquisition when saccharin was used as the target flavor and a different flavor (vinegar) served as the control CS, while no slowreacquisition effect was obtained with vinegar as the target CS.

Experiment 3 tested the generality of the effect obtained in Experiment 2 by using a coffee-flavored solution as the target CS and saccharin as the control flavor. Because on Day 1 subjects consumed small amounts of the $3 \%$ coffee solution used in the previous experiments, and because differences in consumption between saccharin and coffee solutions occurred, a $1 \%$ coffee concentration was used rather than the $3 \%$ coffee solution used in the previous experiments.

\section{Method}

Subjects and Apparatus. Fourteen male Sprague-Dawleyderived rats bred in the University of Missouri colony served as subjects. At the start of the study, the rats weighed 202-304 g. All rats were maintained, and all features of the apparatus were the same, as in Experiment 2, except that a 1\% coffee solution (coff), rather than a $3 \%$ coffee concentration, was used.

Procedure. In Experiment 3, seven animals were assigned to Groups Control and Reacqu, counterbalancing for body weight. On Day 1, the rats were given $10 \mathrm{~min}$ of access to a lick tube containing either coff (Group Reacqu) or sac (Group Control), and this was followed immediately by an injection of $0.3 \mathrm{M} \mathrm{LiCl}$ at $1.33 \%$ body weight. The subjects recovered from illness on Day 2. On Days 3-11, each animal received $10 \mathrm{~min}$ of exposure to the solution to which it had been exposed on Day 1, but without $\mathrm{LiCl}$. No treatments were given on Days 13 and 15, to allow full physiological recovery from the $\mathrm{LiCl}$ treatment to occur. On Days 12 and 14, all subjects received coff for $10 \mathrm{~min}$, followed by an injection of $0.15 \mathrm{M} \mathrm{LiCl}$ at $1 \%$ body weight.

All of the subjects were given 10 min of exposure to coff during testing each day, from Day 16 through Day 25 . All unspecified details of the experiment were unchanged from Experiment 2.

\section{Results and Discussion}

In sum, a slow-reacquisition effect was obtained when the roles of coff and sac in Experiments 1 and 2 were reversed. Two subjects from Group Control were eliminated from the study because they failed to consume at least $2 \mathrm{ml}$ of coff on Day 12 (i.e., on their initial exposure to coff). An ANOVA conducted on the data from the Day 1 conditioning trial revealed no group differences $[F(1,10)=1.38]$. Both groups exhibited an aversion, as demonstrated by a decrease in consumption on the initial extinction trial relative to consumption on Day 1 , revealed by a main effect of day $[F(1,10)=$ 52.06]. There was no effect of group, nor was there a group $\times$ day interaction $[F \mathbf{s}(1,10)<1.62]$. An ANOVA conducted on the extinction data from Phase 2 yielded a main effect of day, showing that the groups increased their consumption across extinction trials $[F(1,10)=$ 17.55]. There was no main effect of group, nor was any interaction found for these data $(F \mathrm{~s}<1)$.

There was no difference in the amount of coff consumed during the coff- $\mathrm{LiCl}$ trial following extinction on Day $12[F(1,10)=1.91, p=.20]$. On Day 12, the groups consumed $9.3( \pm 1.8) \mathrm{ml}$ (Group Reacqu) and $6.0( \pm 1.2) \mathrm{ml}$ (Group Control). This lack of a difference on the Day 12 conditioning trial shows that if any neophobia occurred for Group Control, it did not produce statistically significant lower consumption by that group than by Group Reacqu.

Group mean consumption of coff on the conditioning trial on Day 14 revealed the effect of the conditioning trial on Day 12. Group Reacqu consumed $5.3( \pm 1.4) \mathrm{ml}$ and Group Control drank $0.6( \pm 0.2) \mathrm{ml}$ on Day 14 . This difference was statistically significant $[F(1,10)=8.71$, $p<.02$ ].

The test data from the fifth test trial on Day 20 were lost due to an apparatus problem. Consumption of coff on the test trials on Days 16-19 and 21-25 is represented in Figure 2. There was very low within-group variability for Group Control. Even on Trial 8 when a slight increase in drinking occurred, the scores for this group ranged from 1.7 to $2.3 \mathrm{ml}$. An ANOVA (group $\times$ day) conducted on consumption during the test trials repre-

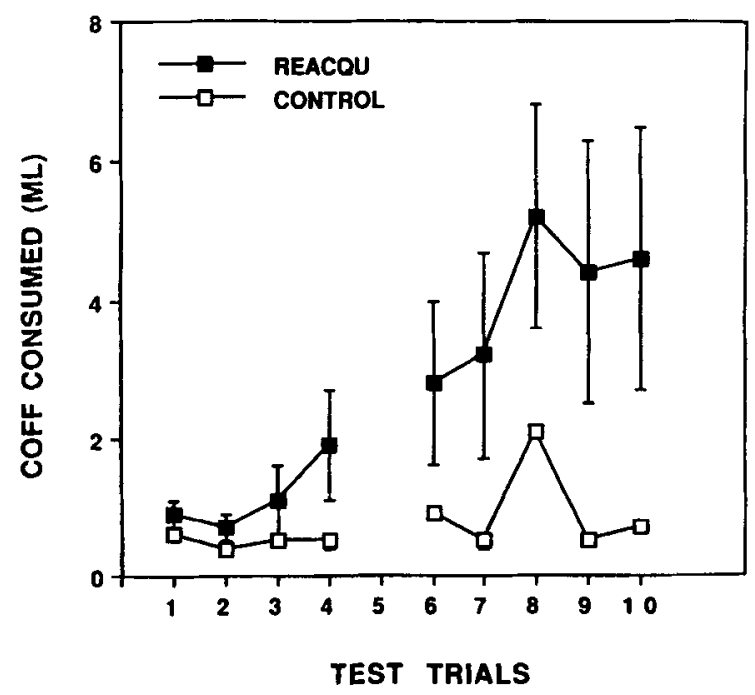

Figure 2. Mean intake ( $\pm S E M s$ ) of a coffee solution on the test trials in Experiment 3. The data from the fifth test trial were not available. 
sented in Figure 2 yielded no main effect of group $[F(1,10)=2.52]$, although a group $\times$ day interaction occurred $[F(8,80)=2.53, p<.02]$. The groups increased their consumption across days $[F(8,80)=5.10]$. These results confirm the slow-reacquisition effect obtained in Experiment 2. The retarded reacquisition of conditioning found in Experiment 2 was not specific to the use of saccharin as the target flavor and a coffeeflavored solution as the control flavor.

\section{GENERAL DISCUSSION}

The present experiments demonstrated slow reacquisition of an extinguished CS in conditioned taste aversion. Slow reacquisition is therefore not specific to the CER procedure of Bouton (1986) and Bouton and Swartzentruber (1989). The results support Danguir and Nicolaidis's (1977) finding of slow reacquisition with a CTA procedure, while conflicting with Revusky and Coombes's (1979) claim that slow reacquisition does not occur with a CTA procedure.

Before discussing the implications of the present findings, we will make a few comments about the procedural features of the present experiments. The experiments used a control condition similar to that used by Bouton in his CER experiments (e.g., Bouton, 1986). This control group received initial conditioning and extinction with a CS that was irrelevant to the treatments given during Phase 3 conditioning and testing. This is a rigorous control condition compared with that of Napier et al.'s (1992) control group that received no conditioning or extinction in the initial phases of the experiment; during the initial phases of the present experiments, the control groups received experience with the LiCl US and with many features of the apparatus, such as drinking from the drinking tubes. Nonetheless, it should be pointed out that previous studies have shown that conditioning with an irrelevant CS (such as the treatment given to the present control groups) can result in transfer of the associative strength from this $\mathrm{CS}$ to a target $\mathrm{CS}$ when the latter CS is paired with the US (Kehoe, Morrow, \& Holt, 1984; Westbrook \& Homewood, 1982). Such transfer would facilitate conditioning in the control group. However, Westbrook and Homewood found, using a CTA procedure, that extinction of the conditioned irrelevant CS prior to conditioning of the target CS can attenuate this transfer (but see Kehoe et al., 1984). Given that the present procedure involved extensive extinction of the control flavor prior to target conditioning, any such transfer of associative strength from the control CS for Group Control was unlikely, and the present control groups provide useful comparison conditions for assessing slow reacquisition.

Some potential role of neophobia in producing the present results cannot be denied, but it is likely that, at most, it exerted a very small effect on the results. In Experiments 2 and 3, the group differences emerging during the second conditioning trial of the later conditioning phase were much greater than those on the first conditioning trial of this phase. In contrast, the greatest neophobia would be expected on the first trial for Group Control. Neophobia is typically greater during the initial exposure to a flavor (e.g., Miller \& Holtzman, 1981), suggesting that the group difference on the second trial of the later conditioning phase was a result of factors other than neophobia. Additionally, group differences during testing were greater on later test trials than they were on early test trials. Neophobia would be expected to exert its greatest influence during the initial exposures to sac of Group Control. The present study could have included an experimental group that received identical experience with the target solution during all phases of the experiments, except for not receiving the original conditioning with this CS in Phase 1-in other words, it could have included a control group that received extensive CS preexposure (i.e., latent inhibition) prior to the Phase 3 conditioning phase. This control group would be roughly equated with the experimental group for exposure to the target CS. Bouton and Swartzentruber (1989) included such a control and found, not surprisingly, that latent inhibition produced even slower reacquisition than that demonstrated by the group that received identical treatment except for also receiving the CS paired with the US on Day 1. A similar effect would have been expected if such a group had been included in the present study.

Conditioning is usually very rapid in taste-aversion conditioning, thereby producing almost complete suppression of drinking after one or two training trials. To test the associative strength of the CS, several test trials were given to observe group differences. These tests essentially constituted a reextinction treatment. It is usually assumed that the rate of extinction is inversely correlated with the strength of the CS-US association; yet, rather than interpreting the present results as reflecting slow reconditioning, one could view them as reflecting rapid reextinction by Group Reacqu. The present findings, however, argue against this claim. In Experiments 2 and 3 , pronounced group differences were found during consumption on the second conditioning trial during the second conditioning phase of the study. This trial can be seen as a test trial that reflects the learning occurring on the first conditioning trial of the postextinction conditioning phase. These effects are not amenable to an explanation based on reextinction rate.

The agreement of the present results with those of Danguir and Nicolaidis (1977), but not with those of Revusky and Coombes (1979), raises concern about why the results differ between the studies. One factor that probably contributed to Revusky and Coombes's failure to obtain slow reacquisition in at least some of their experiments is that their control groups received extensive preexposure (e.g., 10 exposures in some experiments and 8 in another experiment) to the flavor that was to be conditioned just prior to testing. It is likely that this CS-alone preexposure produced substantial latent inhibition for the control subjects, thereby greatly retarding acquisition and attenuating any opportunity to observe slower reacquisition among the experimental subjects. 
These control treatments used by Revusky and Coombes make it unsurprising that a savings effect was found with saccharin in one of their experiments. Danguir and Nicolaidisis's control groups also received extensive nonreinforced preexposure to the to-be-conditioned saccharin flavor, making their finding of retarded reacquisition surprising, but especially convincing.

The present results provide information about the types of associations that are susceptible to slow reacquisition. Napier et al. (1992) discussed the possibility that slow reacquisition is dependent on the type of conditioning procedure used. As mentioned earlier, retarded reacquisition is not obtained with an NMR procedure with rabbits, while slow reacquisition does occur with CTA and CER procedures with rats. Napier et al. suggested that different conditioning procedures may activate different response systems. Konorski (1967) proposed two response systems-namely, preparatory and consummatory - that may operate during the formation of associations. Preparatory responses include behaviors that reflect expression of emotional states, such as heartrate changes and overall motor activity. Consummatory responses involve behaviors directed at the US, such as pecking, salivating, and the blink response of NMR conditioning. If slow reacquisition occurs for preparatory but not consummatory responses, and if CTA is considered a preparatory response, the disparate results can be explained using this framework. It is likely that CTA is an instance of preparatory responding, and such avoidance behaviors were described by Konorski as part of the preparatory response system (Konorski, 1967, p. 29).

The question of whether extinction produces slow reacquisition has been used to dissociate models of conditioning, including tests of CS-effectiveness models (Lubow, Weiner, \& Schnur, 1981; Pearce \& Hall, 1980; Wagner, 1978), as well as connectionist models (Kehoe, 1988; Klopf, 1988). CS-effectiveness models claim that a CS can lose its potential to change its associative value (i.e., that it can lose CS effectiveness) when it is presented alone during extinction. The present demonstrations of slow reacquisition are readily explained by CSeffectiveness models.

In contrast with CS-effectiveness explanations of extinction and slow reacquisition, the interference view states that retroactive interference produces the poor $\mathrm{CR}$ that results from extinction (e.g., Bouton, 1993; Kraemer \& Spear, 1992; Miller, Kasprow, \& Schachtman, 1986; Spear, 1978). During conditioning, subjects learn a CS-US association. During extinction, subjects learn a CS-no-US association, and the latter learning interferes with retrieval of the CS-US association; hence, a poor CR occurs at test. This view has been supported by numerous findings, including, for example, the effects of US reinstatement, renewal effects, and slow reacquisition (i.e., Bouton, 1986; Bouton \& Peck, 1989; Bouton \& Swartzentruber, 1989; Schachtman et al., 1985; Schachtman, Gustavson, Chelonis, \& Bourne, 1992). The CS-noUS association can also act proactively to slow the reac- quisition of the $\mathrm{CR}$ during the reconditioning phase and produce the slow-reacquisition effect (Bouton, 1993). The present results are consistent with an interference view of extinction. Extensive extinction produces strong CS-no-US associations that yield greater interference with retrieval and expression of the CS-US association.

In addition to potentially producing interference and/or changes in CS effectiveness, extensive extinction may also endow a stimulus with conditioned inhibitory properties. Several early and recent learning theories have claimed that extinction can provide an excitatory CS with inhibitory properties (Hull, 1943; Konorski, 1948; Pavlov, 1927; Pearce \& Hall, 1980; Wagner, 1978). Recent experiments in our laboratory demonstrated that an extensively extinguished CS cannot only "pass" a retardation test for conditioned inhibition (i.e., show slow reacquisition), it can pass a summation test as well. There are a number of potential processes underlying extinction of a conditioned excitor. It remains to be determined whether extinction treatments produce one such process, or whether they result in several different effects.

\section{REFERENCES}

BouTon, M. E. (1986). Slow reacquisition following extinction of conditioned suppression. Learning \& Motivation, 17, 1-15.

Bouton, M. E. (1993). Context, time, and memory retrieval in the interference paradigms of Pavlovian learning. Psychological Bulletin, $114,80-99$.

Bouton, M. E., \& PeCK, C. A. (1989). Context effects on conditioning, extinction, and reinstatement in an appetitive conditioning preparation. Animal Learning \& Behavior, 17, 188-198.

Bouton, M. E., \& Swartzentruber, D. A. (1989). Slow reacquisition following extinction: Context, encoding, and retrieval mechanisms. Journal of Experimental Psychology: Animal Behavior Processes, 15, 43-53.

DANGUIR, J., \& Nicolaidis, S. (1977). Lack of reacquisition in learned taste aversions. Animal Learning \& Behavior, 5, 395-397.

Hull, C. L. (1943). Principles of behavior. New York: AppletonCentury-Crofts.

KEHOE, E. J. (1988). A layered network model of associative learning: Learning-to-learn and configuration. Psychological Review, 95, 411-433.

KeHOE, E. J., MORROW, L. D., \& HoLT, P. E. (1984). General transfer across sensory modalities survives reductions in the original conditioned reflex in the rabbit. Animal Learning \& Behavior, 12, 129-136.

KLOPF, A. H. (1988). A neuronal model of classical conditioning. Psychobiology, 16, 85-125.

KoNORSKI, J. (1948). Conditioned reflexes and neuron organization. Cambridge: Cambridge University Press.

KoNORSKI, J. (1967). Integrative activity of the brain. Chicago: University of Chicago Press.

Kraemer, P. J., \& SPEAR, N. E. (1992). The effect of nonreinforced stimulus exposure on the strength of a conditioned taste aversion as a function of retention interval: Do latent inhibition and extinction involve a shared process? Animal Learning \& Behavior, 20, 1-7.

LUBOW, R. E., WEINER, I., \& SCHNUR, P. (1981). Conditioned attention theory. In G. H. Bower (Ed.), The psychology of learning and motivation (Vol. 15, pp. 1-49). San Diego, CA: Academic Press.

Miller, R. R., \& Holtzman, A. D. (1981). Neophobia: Generality and function. Behavioral \& Neural Biology, 33, 17-44.

Miller, R. R., Kasprow, W. J., \& Schachtman, T. R. (1986). Retrieval variability: Sources and consequences. American Journal of Psychology, 99, 145-218

Napier, R. M., Macrae, M., \& Kehoe, E. J. (1992). Rapid reacquisition in conditioning of the rabbit's nictitating membrane response. 
Journal of Experimental Psychology: Animal Behavior Processes, 18, 182-192.

Pavlov, I. P. (1927). Conditioned reflexes: An investigation of the physiological activity of the cerebral cortex (G. V. Anrep, Ed. and Trans.). London: Oxford University Press.

Pearce, J. M., \& Hall, G. (1980). A model for Pavlovian learning: Variations in the effectiveness of conditioned but not of unconditioned stimuli. Psychological Review, 94, 61-73.

Revusky, S., \& CoOmbes, S. (1979). Reacquisition of learned taste aversions. Animal Learning \& Behavior, 7, 377-382.

Schachtman, T. R., Brown, A. M., \& Miller, R. R. (1985). Reinstatement-induced recovery of a taste- $\mathrm{LiCl}$ association following extinction. Animal Learning \& Behavior, 13, 223-227.

Schachtman, T. R., Gustavson, K. K., Chelonis, J. J., \& Bourne,
M. J. (1992). Effects of US reinstatement on the potential of an extinguished CS to attenuate manifest learning about another CS. Learning \& Motivation, 23, 250-268.

SPEAR, N. E. (1978). The processing of memories: Forgetting and retention. Hillsdale, $\mathrm{NJ}$ : Erlbaum.

WAGNER, A. R. (1978). Expectancies and priming of STM. In S. H. Hulse, H. Fowler, \& W. K. Honig (Eds.), Cognitive processes in animal behavior (pp. 177-209). Hillsdale, NJ: Erlbaum.

WestBRook, R. F., \& Homewood, J. (1982). The effects of a flavourtoxicosis pairing upon long-delay, flavour-aversion learning. Quarterly Journal of Experimental Psychology, 34B, 59-75.

(Manuscript received August 25, 1993; revision accepted for publication August 5, 1994.) 